\title{
Magnetoimpedance Response and Field Sensitivity in Stress-Annealed Co-Based Microwires for Sensor Applications
}

\author{
David González-Alonso ${ }^{1}$, Lorena González-Legarreta ${ }^{2,3, *}$, Paula Corte-León ${ }^{3,4}$, \\ Valentina Zhukova ${ }^{3,4}\left(\mathbb{D}\right.$, Mihail Ipatov ${ }^{3,4}$, Juan María Blanco ${ }^{4}$ and Arcady Zhukov ${ }^{3,4,5, *(D)}$ \\ 1 Departmento CITIMAC, Facultad de Ciencias, Universidad de Cantabria, E-39005 Santander, Spain; \\ david.gonzalezalonso@unican.es \\ 2 Departmento QUIPRE, Inorganic Chemistry-University of Cantabria, Nanomedice-IDIVAL, \\ Avda. de Los Castros 46, 39005 Santander, Spain \\ 3 Departmento de Física de Materiales, Facultad de Químicas, Universidad del País Vasco/Euskal Herriko \\ Unibersitatea, UPV/EHU, Paseo Manuel de Lardizabal, 3, 20018 San Sebastián, Spain; \\ paula.corte@ehu.eus (P.C.-L.); valentina.zhukova@ehu.es (V.Z.); mihail.ipatov@ehu.es (M.I.) \\ 4 Departmento de Física Aplicada, EIG, Universidad del País Vasco/Euskal Herriko Unibersitatea, UPV/EHU, \\ 20018 San Sebastian, Spain; juanmaria.blanco@ehu.es \\ 5 IKERBASQUE, Basque Foundation for Science, 48011 Bilbao, Spain \\ * Correspondence: gonzalezll@unican.es (L.G.-L.); arkadi.joukov@ehu.es (A.Z.)
}

Received: 17 May 2020; Accepted: 3 June 2020; Published: 5 June 2020

\begin{abstract}
Amorphous soft magnetic microwires have attracted much attention in the area of sensor applications due to their excellent properties. In this work, we study the influence of annealing treatments (stress and conventional) in the giant magnetoimpedance (GMI) response and the field sensitivity of the soft magnetic $\mathrm{Co}_{69.2} \mathrm{Fe}_{3.6} \mathrm{Ni}_{1} \mathrm{~B}_{12.5} \mathrm{Si}_{11} \mathrm{Mo}_{1.5} \mathrm{C}_{1.2}$ glass-coated microwires. Here we report a remarkable and simultaneous enhancement of GMI effect and field sensitivity. The highest sensitivity of $104 \% / \mathrm{Oe}$ and the GMI response of $234 \%$ were achieved for $300{ }^{\circ} \mathrm{C}$ stress-annealed samples at 472 and $236 \mathrm{MPa}$, respectively. Additionally, we found that stress-annealed microwires exhibit a frequency dependence on maximal GMI response and field sensitivity. These findings are obtained by fine-tuning their magnetoeslastic anisotropies through stress-annealing treatments of as-prepared microwires at the proper temperature and axial applied stress upon annealing. We hope that the results presented here widen the scope of investigations for the future design of soft magnetic materials for sensor purposes.
\end{abstract}

Keywords: magnetoimpedance effect; skin effect; soft magnetic materials; field sensitivity; amorphous microwires

\section{Introduction}

Over the last decades, magnetic sensors have captivated scientific attention for their technological findings in a broad scope of fields. These applications range from space research, military applications, security systems, high-density magnetic recording and biomedicine [1-6]. In this line, the miniaturization of sensors and technological devices opens new routes in the property-to-function conversion. Metal-based amorphous alloys $[7,8]$ constitute the main family within magnetic sensors. After Panina et al. [7,8], Co-based rapidly quenched materials have become widely recognized magnetic materials for sensors due to their high-performance properties $[9,10]$. It should be highlighted that their excellent magnetic softness, exhibiting nearly zero magnetostriction constant value and a 
remarkably giant magnetoimpedance (GMI) response along with high field sensitivity, among other properties [7,11,12].

In this sense, giant magnetoimpedance (GMI) effect results is the most remarkable feature for sensor applications. This phenomenon consists in a significant change of the AC impedance in presence of a static dc-field [13]. GMI ratios up to $600 \%$, at frequencies around $10 \mathrm{MHz}$ [14] and sensitivities up to $10 \% / \mathrm{Am}^{-1}$ are the maximum values reported to date for amorphous wires [15]. These properties make Co-based materials quite suitable candidates for GMI technology, such as magnetic field sensors integrated in complementary metal-oxide-semiconductor (CMOS) circuits [16], high sensitive magnetometers [17] or biomagnetic field detection [18,19].

Co-based glass-coated microwires are attracting scientific interest for their potential in the development of small-scale sensors based on their tunable magnetic properties. The Co-based microwires investigated in this work are fabricated by the Taylor-Ulitovsky technique $[20,21]$. The Taylor-Ulitovsky technique involving rapid solidification of metallic alloys inside the glass coating allows the preparation of long (up to $10 \mathrm{~km}$ ) continuous composite metallic microwires coated by a flexible and insulating glass cover (typical thickness of $0.5-20 \mu \mathrm{m}$ ). At suitable fabrication conditions, a completely amorphous structure with no trace of crystalline phases can be obtained [20,21]. Magnetocrystalline anisotropy contribution is consequently negligible, while their magnetic properties are principally governed by the magnetoelastic interactions. Furthermore, the magnetoelastic interactions play a key role in the GMI response $[15,21,22]$. In fact, these interactions are determined by both the magnetostriction coefficient and internal stresses [23]. Apart from these contributions, classical electrodynamics gives the fundamental support to explain satisfactorily the GMI effect by considering the skin effect of a magnetic conductor [24]. Based on this assumption, the most relevant pre-requisite to achieve a giant MI response is to design amorphous microwires with significant circumferential magnetic permeability, which can be reached by fine-tuning their magnetic properties [25-27].

With respect to the magnetoelastic anisotropy of glass-coated microwires, the magnetostriction coefficient, $\lambda_{\mathrm{s}}$, is dictated by the chemical composition (at fixed internal stresses values which in turn are affected by the fabrication conditions) [28]. Thus, the replacement of Fe by Co atoms permits the adjustment of $\lambda_{\mathrm{s}}$-values from positive (typically $\lambda_{\mathrm{s}} \sim 40 \times 10^{-6}$ for Fe-based microwires) to negative (about $\lambda_{\mathrm{s}} \sim-5 \times 10^{-6}$ for Co-based microwires) values [28,29]. Accordingly, the vanishing $\lambda_{\mathrm{s}}-$ values can be achieved in Co-Fe or Co-Mn amorphous alloys when the content of $\mathrm{Co} / \mathrm{Fe}$ or $\mathrm{Co} / \mathrm{Mn}$ is about $70 / 5$ [28,29]. Moreover, for alloys with vanishing $\lambda_{s}-$ values the stresses influence can be relevant [28]. On the other hand, the internal stresses (value and distribution), which arise from the fabrication of glass-coated microwires, are the other factor affecting the magnetoelastic anisotropy $[15,21,23]$. The internal stresses, $\sigma_{i}$, value can be caused by: i) the thermal expansion coefficient mismatch between metallic-core and the glass-coating; ii) the quenching stresses originated during the rapid solidification process; and iii) the drawing stresses [30,31]. According to most theoretical estimations, the internal stresses arising from the thermal expansion coefficient mismatch are expected to present the largest contribution [30,31].

In this regard, at a fixed composition and geometry (metallic nucleus diameter and glass-coating thickness) the magnetic anisotropy can be fine-tuned either by the internal stresses relaxation (usually by conventional annealing) or by inducing magnetic anisotropy [27,32]. However, it has been recently noticed that conventional annealing produces a considerable magnetic hardening in different Co-rich microwires with vanishing $\lambda_{\mathrm{s}}$-values $[32,33]$. Such magnetic hardening is a detriment to the GMI performance $[27,32,33]$. Nevertheless, we have recently observed that stress-annealing can be successfully employed to induce a transverse anisotropy and hence improve the GMI effect of magnetic microwires $[27,32,33]$.

For those reasons, our aim is to identify the routes that allow the optimization of GMI performance at a fixed chemical composition and geometry (i.e., with fixed $\lambda_{\mathrm{S}}$ and $\sigma_{i}$ values). We expect that in the future the observed dependencies can be extended to various Co-rich microwires. 
Accordingly, in this work, we have investigated the magnetic properties of Co-based amorphous microwires with a well-established chemical composition under stress-annealing conditions aiming to simultaneously enhance their GMI response and magnetic field sensitivity within the range $10 \leq f \leq 1000 \mathrm{MHz}$ of intermediate frequencies.

\section{Experimental Methods}

The $\mathrm{Co}_{69.2} \mathrm{Fe}_{3.6} \mathrm{Ni}_{1} \mathrm{~B}_{12.5} \mathrm{Si}_{11} \mathrm{Mo}_{1.5} \mathrm{C}_{1.2}$ microwire consists in a metallic nucleus of $\mathrm{d}=22.8 \mu \mathrm{m}$ diameter, surrounded by an outer glass-coating shell that results in $\mathrm{D}=23.2 \mu \mathrm{m}$ total diameter. The amorphous microwire has been fabricated using the Taylor-Ulitovsky technique, which is described elsewhere [20,21]. As in similar Co-based microwires [28], the sample presents a slightly negative magnetostriction coefficient $\lambda_{S} \approx-1 \times 10^{-7}$ [27]. This allows us to predict the magnetic softness in our sample and suggests the existence of a circumferential anisotropy along the microwire. This latter feature is the underlying ingredient to obtain remarkable MI response [24,34].

For proper modification to the magnetic properties of our sample, slices of the as-prepared microwire were subsequently heat-treated in a standard furnace by two different methods. In one method the annealing is performed with no applied stress, whereas in the second it is conducted under axial tensile stress. The tensile stress, $\sigma_{m}$, was applied through a mechanical load attached to the end of the microwire and axially placed via the furnace nozzle, allowing stresses up to $472 \mathrm{MPa}$. The $\sigma_{m}$-values are evaluated considering the different Young's moduli of the metallic nucleus and the glass coating as recently described [33]. All these treatments were performed at selected temperatures, $T_{a n n}$, ranged from 200 to $400{ }^{\circ} \mathrm{C}$ and for $1 \mathrm{~h}$ duration, $t_{a n n}$. This annealing temperature range has been selected considering the onset of the crystallization process reported at ca. $490{ }^{\circ} \mathrm{C}$ for microwires of the same composition [35]. In fact, it has been described that the rising of the annealing temperature close to the crystallization point causes a deterioration of both mechanical and magnetic properties in Co-rich microwires [36,37]. Although two of the advantages of glass-coated microwires are their flexibility and insulating properties of the glass coating, an excess of temperature can damage the outer glass-coating.

Magnetic hysteresis loops (HLs) were measured using the fluxmetric method already described [33]. Microwire slices of $5 \mathrm{~cm}$ in length were placed inside a single layered pick-up coil, where a magnetic field was created by a $15 \mathrm{~cm}$ long solenoid. For better comparison of the treated microwires, HLs are represented as the normalized magnetization $M / M_{0}$, where $M$ is the measured magnetic moment at a given magnetic field, and $M_{0}$ is the maximal magnetic moment obtained at the highest magnetic field amplitude $H_{\max }$.

Impedance measurements, Z, were carried out with a vector network analyzer (VNA) N5230A at room temperature. Microwires of $6 \mathrm{~mm}$ length were fixed to a micro-strip sample holder by tin soldering, and subsequently placed inside a long solenoid that creates a maximum homogeneous magnetic field, $H$, of $15 \mathrm{kA} / \mathrm{m}$ (ca. $189 \mathrm{Oe}$ ). Z-values are indirectly obtained in the intermediate frequency range $10-1000 \mathrm{MHz}$ through the measurement of the reflection coefficient $\mathrm{S}_{11}$, using the following expression [34,38]:

$$
\mathrm{Z}=\mathrm{Z}_{0} \frac{\left(1+S_{11}\right)}{\left(1-S_{11}\right)}
$$

where $Z_{0}=50 \mathrm{Ohm}$ is the characteristic impedance of the coaxial line.

The MI response or GMI ratio, $\Delta \mathrm{Z} / \mathrm{Z}$, is determined from $\mathrm{Z}$-values, which are obtained for different magnetic fields measurements, and it is defined as:

$$
\Delta \mathrm{Z} / \mathrm{Z}=\frac{\left[\mathrm{Z}(H)-\mathrm{Z}\left(H_{\max }\right)\right]}{\mathrm{Z}\left(H_{\max }\right)} \times 100
$$

where $H_{\max }$ is the highest dc-magnetic field applied. 
A distinctive feature of MI response to discriminate among magnetic sensor materials is the field sensitivity, $\eta$, which is calculated through:

$$
\eta=\frac{\partial\left(\frac{\Delta Z}{Z}\right)}{\partial H}
$$

\section{Results and Discussion}

The MI response in amorphous ferromagnetic microwires can be enhanced by relaxing their inner stresses through a diversity of thermal treatments. In view of this, we present here the advantages of stress-annealing when optimising the magnetic properties of the $\mathrm{Co}_{69.2} \mathrm{Fe}_{3.6} \mathrm{Ni}_{1} \mathrm{~B}_{12.5} \mathrm{Si}_{11} \mathrm{Mo}_{1.5} \mathrm{C}_{1.2}$ as-prepared microwire, in contrast to conventional annealing. Figure 1a shows the HLs of the as-prepared, along with the annealed microwires performed at selected temperatures $T_{\text {ann }}$ below its crystallization point $\left(\sim 550^{\circ} \mathrm{C},[35]\right)$. In the inset of Figure 1a, it is observed a magnetic hardening after $1 \mathrm{~h}$ annealing. Particularly, in Figure 2a we depict the effect of conventional annealing by increasing the $H_{c}$ from ca. $0.06 \mathrm{Oe}(5 \mathrm{~A} / \mathrm{m})$ for the as-prepared sample, up to ca. $1.26 \mathrm{Oe}(100 \mathrm{~A} / \mathrm{m})$ for the conventional annealed microwire at the highest $T_{\text {ann }}$ of $400{ }^{\circ} \mathrm{C}$.
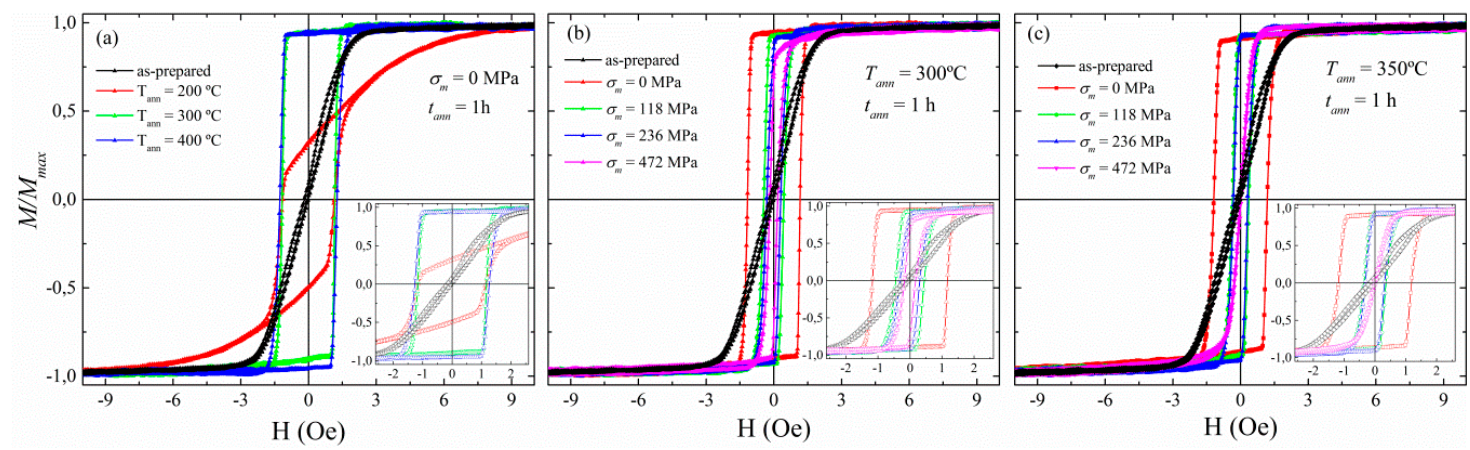

Figure 1. (a) Hysteresis loops (HLs) of the as-prepared and the annealed microwires performed at $200{ }^{\circ} \mathrm{C}, 300{ }^{\circ} \mathrm{C}$, and $400{ }^{\circ} \mathrm{C}$. Subfigures (b) and (c) show HLs of as-prepared and stress-annealed performed at $T_{a n n}=300$ and $350{ }^{\circ} \mathrm{C}$, respectively. The thermal treatments on the parent-microwire $\mathrm{Co}_{69.2} \mathrm{Fe}_{3.6} \mathrm{Ni}_{1} \mathrm{~B}_{12.5} \mathrm{Si}_{11} \mathrm{Mo}_{1.5} \mathrm{C}_{1.2}$ were performed for $1 \mathrm{~h}$. In the insets, it is shown a magnification of the HLs to make clear the linear-to-rectangular evolution of the treated microwires.

The effect of conventional annealing on HLs of Co-rich microwires with low-negative magnetostriction $\lambda_{s}$ has already been studied [23]. Conventional annealing is expected to produce magnetic hardening, along with an increment in the magnetization of the metallic nucleus as evinced in the linear-to-rectangular evolution of the HLs in Figure $1 \mathrm{a}$ as $T_{\text {ann }}$ rises. The magnetic hardening is explained through internal stresses relaxation that brings about circumferential domain structure along the microwire. This hardening could also be the result of either a growth of inner axially magnetized domains [21,26,38], or a variation in the magnetostriction value [39,40], or even a sign change in the magnetostriction coefficient $[23,40,41]$.

Hence, conventional annealing cannot be considered the best post-processing treatment to improve magnetic softness of Co-based microwires. In this regard, stress-annealing counteracts the magnetic hardening experienced by the microwire on conventional annealing. This effect is plainly visible in the insets of Figure 1b,c, where two characteristic stress-annealing temperatures are represented, $T_{\text {ann }}=300$ and $350^{\circ} \mathrm{C}$, respectively. There, the coercive field, $H_{c}$, is reduced while achieving a magnetic softening, which is more clearly shown in Figure $2 \mathrm{~b}$. In addition, microwire soft magnetic properties can be affected by the stress-annealed conditions, i.e., $T_{a n n}, t_{a n n}$ and $\sigma_{m}[27,33,40,41]$. Figure $1 c$ shows that the linear HLs typically observed for the as-prepared microwire are recovered at high enough $T_{a n n}$ and $\sigma_{m}$ (in violet), but at the expense of $M_{r} / M_{0}$-values (where $M_{r}$ is the remanent magnetization). This decrease in the $M_{r} / M_{0}$ ratio results in a reduction of the GMI response as is observed below for the 
stress-annealed microwire at $T_{a n n}=300^{\circ} \mathrm{C}$ and $\sigma_{m}=472 \mathrm{MPa}$. These changes of magnetic properties result from an increase in circumferential magnetic anisotropy, which is induced by stress-annealing and becomes more significant when increasing $T_{a n n}, t_{\text {ann }}$ and $\sigma_{m}[33,38]$.
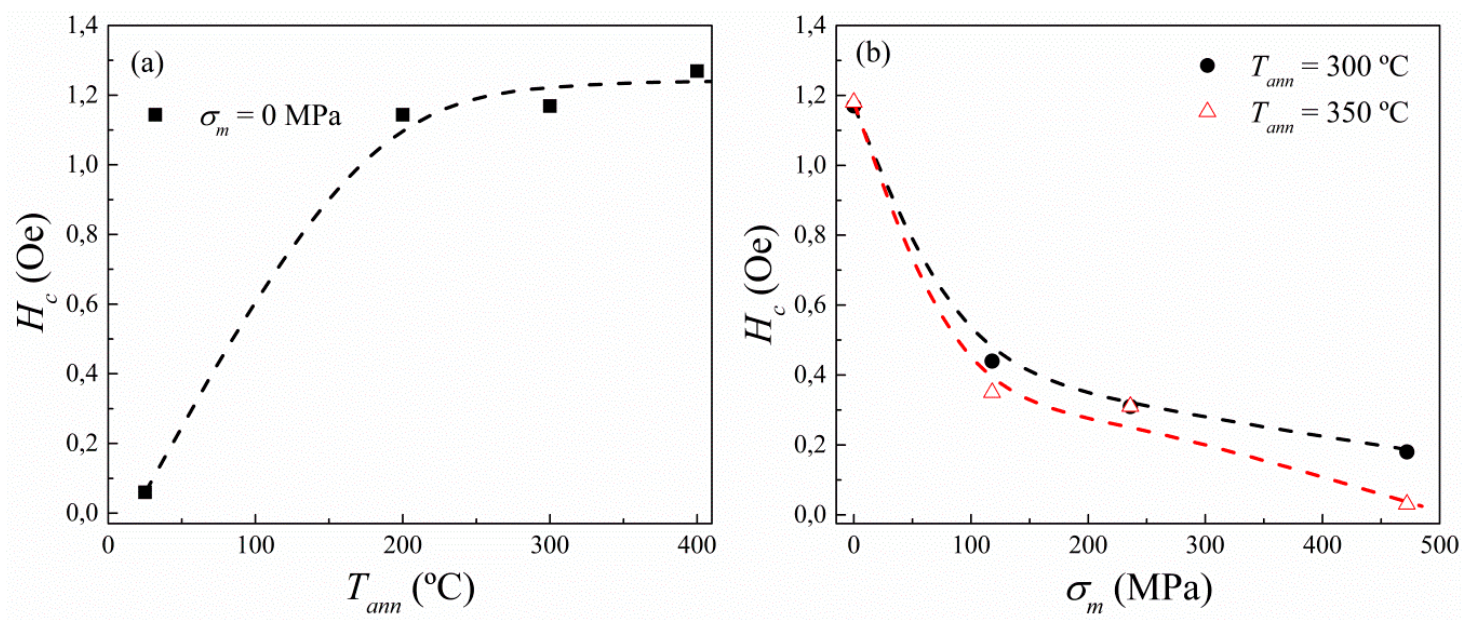

Figure 2. Temperature-annealing (a) and stress-annealing (b) dependence on the $H_{c}$ of $\mathrm{Co}_{69.2} \mathrm{Fe}_{3.6} \mathrm{Ni}_{1} \mathrm{~B}_{12.5} \mathrm{Si}_{11} \mathrm{Mo}_{1.5} \mathrm{C}_{1.2}$ microwires for $1 \mathrm{~h}$ of thermal-treatment. Dashed lines are a guide to the eyes.

In Figure 3 we confirm the dissimilar effect of conventional annealing on the GMI response depending on $T_{a n n}$. On the one hand, Figure 3 a shows a reduction in the GMI ratio upon annealing at $200{ }^{\circ} \mathrm{C}$, decreasing from $103 \%$ (for the as-prepared) down to $62 \%$ at $100 \mathrm{MHz}$. However, a clear improvement in the GMI effect is achieved when annealing at $300{ }^{\circ} \mathrm{C}$, rising from $103 \%$ up to $141 \%$ at $100 \mathrm{MHz}$. Furthermore, despite the opposite effect of conventional annealing on the GMI response, it is noteworthy that in Figure $3 c$ the maximum value of the $\Delta Z / Z_{\text {max }}$ ratio is monotonously shifted to high-frequencies as the $T_{\text {ann }}$ rises. This characteristic frequency, hereinafter $f_{\text {char }}$, is defined as the frequency at which the $\Delta Z / Z_{\max }$ ratio is maximal. In addition, in Figure $3 \mathrm{~d}$ the field sensitivity displays minor differences in the frequency dependence between samples. Therefore, conventional annealing is revealed as an adequate treatment to improve the GMI response only at certain annealing conditions.

With regard to the effect of stress-annealing on the GMI response, in Figure 4 it is clearly appreciated as a significant GMI improvement as compared to conventional annealing for all stress-annealed microwires. For example, the measured $\Delta Z / Z$ ratio of the as-prepared microwire is equal to $103 \%$ at $100 \mathrm{MHz}$, whereas an increase up to $166 \%$ is found in the stress-annealed microwire at $200{ }^{\circ} \mathrm{C}$ and $118 \mathrm{MPa}$ (see Figure $4 \mathrm{a}$ ). By contrast, a reduction in the $\Delta \mathrm{Z} / \mathrm{Z}$ ratio down to $62 \%$ is observed in Figure $3 \mathrm{a}$ for the conventional annealed microwire at $200{ }^{\circ} \mathrm{C}$. The improvement in the GMI response clearly is stated in Figure 4c for the whole frequency range (up to $1000 \mathrm{MHz}$ ). Moreover, Figure 4c shows a slight increase in $f_{\text {char }}$ up to ca. $150 \mathrm{MHz}$ for the $\Delta Z / Z_{\text {max }}$ of the stress-annealed microwires, in comparison with the as-prepared microwire where the maximum is centred at ca. $80 \mathrm{MHz}$. Figure $4 \mathrm{~d}$ draws a clear improvement in the field sensitivity for the stress-annealed samples, in contrast to conventional annealing. Particularly, at $100 \mathrm{MHz}$, the field sensitivity rises from 10\%/Oe for the as-prepared, up to $\eta=64 \% / O e$ when stress-annealing at $300^{\circ} \mathrm{C}$ and $118 \mathrm{MPa}$. However, the field sensitivity obtained for the three stress-annealed samples is quite similar in the frequency range $500 \leq f \leq 1000 \mathrm{MHz}$. In fact, they follow the same tendency as those shown in Figure $4 \mathrm{c}$ for the frequency dependence of $\Delta Z / Z_{\text {max }}$. In summary, stress-annealing arises here as the suitable technique to enhance simultaneously the GMI ratio and field sensitivity. 

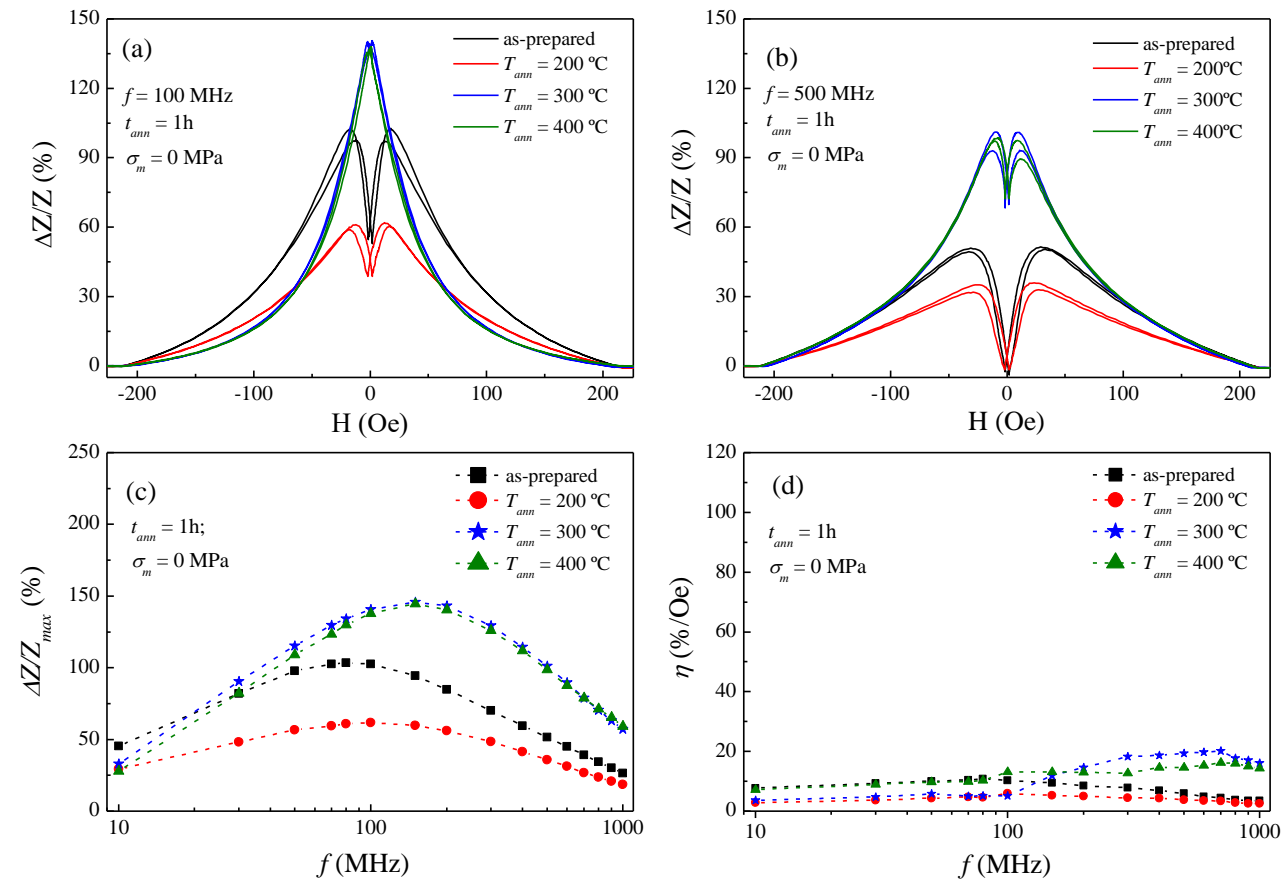

Figure 3. Field dependence of the GMI ratio for the as-prepared and annealed $\mathrm{Co}_{69.2} \mathrm{Fe}_{3.6} \mathrm{Ni}_{1} \mathrm{~B}_{12.5} \mathrm{Si}_{11} \mathrm{Mo}_{1.5} \mathrm{C}_{1.2}$ microwires measured at representative frequencies: (a) $100 \mathrm{MHz}$ and (b) $500 \mathrm{MHz}$; (c) frequency dependence of $\Delta \mathrm{Z} / \mathrm{Z}_{\max }$, and (d) field sensitivity. Dashed-lines in (c) and (d) are a guide to the eye, while full-symbols denote experimental data.
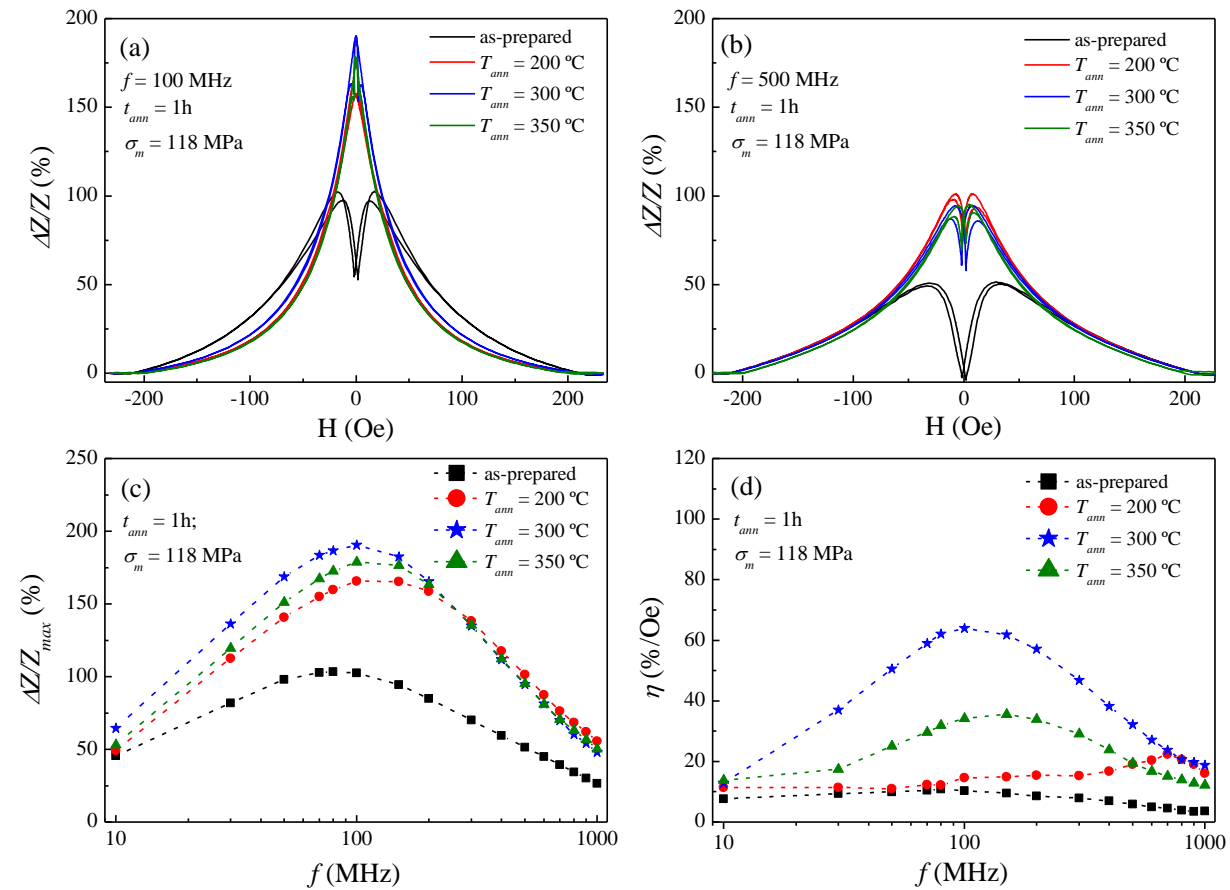

Figure 4. Field dependence of the GMI ratio for the as-prepared and $118 \mathrm{MPa}$ stress-annealed $\mathrm{Co}_{69.2} \mathrm{Fe}_{3.6} \mathrm{Ni}_{1} \mathrm{~B}_{12.5} \mathrm{Si}_{11} \mathrm{Mo}_{1.5} \mathrm{C}_{1.2}$ microwires measured at representative frequencies: (a) $100 \mathrm{MHz}$ and (b) $500 \mathrm{MHz}$; (c) frequency dependence of $\Delta \mathrm{Z} / \mathrm{Z}_{\max }$, and (d) field sensitivity. Dashed-lines in (c,d) are a guide to the eye, while full-symbols denote experimental data.

In this line, the $118 \mathrm{MPa}$ stress-annealed microwire at $300^{\circ} \mathrm{C}$ presents the highest GMI response and field sensitivity, as show in Figure $4 \mathrm{c}, \mathrm{d}$, respectively. For this reason, it was resolved to make 
a more detailed investigation of the stress-annealing effect under different applied stresses, but at a fixed $T_{\text {ann }}$ of $300^{\circ} \mathrm{C}$ (see Figure 5). It is worth noticing the remarkable improvement of both the GMI effect and field sensitivity, though at different stress-annealing treatments. Figure $5 \mathrm{c}$ shows the positive evolution of $\Delta Z / Z_{\max }$ as the applied stress $\sigma_{m}$ rises. In fact, this positive change is even more evident in the field sensitivity response (see Figure 5d). Specifically, at $100 \mathrm{MHz}$ the GMI ratio improves up to $234 \%$ for an applied stress of $236 \mathrm{MPa}$, in contrast to the $103 \%$ for the as-prepared sample. On the other hand, the field sensitivity is enhanced up to $104 \% / \mathrm{Oe}$ for the $472 \mathrm{MPa}$ stress-annealed microwire, while the as-prepared exhibits a poor $10 \% / O e$. Therefore, the field sensitivity improves as the applied stress rises (see Figure $5 \mathrm{~d}$ ) within the following range $10 \mathrm{MHz} \leq f \leq 200 \mathrm{MHz}$. This frequency range is the preferred for sensors applications because of better signal to noise features and hence lower price of electronic circuits, allowing easier processing of the electronic signals [17]. In short, this makes our stress-annealed sample a suitable material for sensor applications [9]. It is worth noting that for other families of thicker Co-rich wires $(\mathrm{d}=120 \mu \mathrm{m})$ prepared by the in-rotating water technique a $f_{\text {char }}<1 \mathrm{MHz}$ has been reported [42].
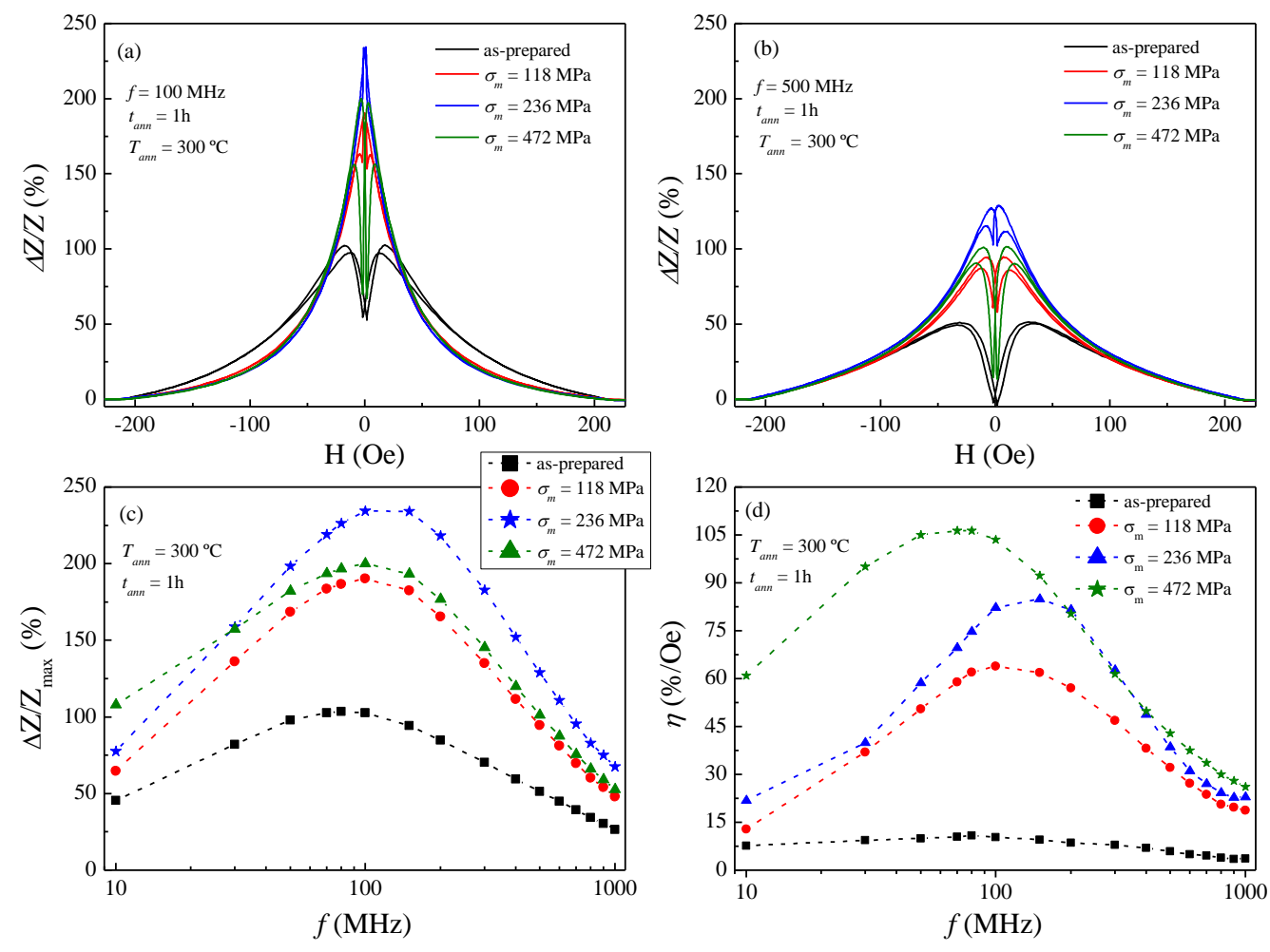

Figure 5. Field dependence of the GMI ratio for the as-prepared and stress-annealed $\mathrm{Co}_{69.2} \mathrm{Fe}_{3.6} \mathrm{Ni}_{1} \mathrm{~B}_{12.5} \mathrm{Si}_{11} \mathrm{Mo}_{1.5} \mathrm{C}_{1.2}$ microwires measured at $300{ }^{\circ} \mathrm{C}$ : (a) $100 \mathrm{MHz}$ and (b) $500 \mathrm{MHz}$; (c) frequency dependence of $\Delta \mathrm{Z} / \mathrm{Z}_{\max }$, and (d) field sensitivity. Dashed-lines in (c,d) are a guide to the eye, while full-symbols denote experimental data.

All the experimental results reported here could be described considering that the origin of the GMI effect is directly connected to the skin depth (ac frequency $f$ ) and the circumferential magnetic permeability (through the external dc magnetic field $H_{d c}$, ac current, and induced anisotropies). Consequently, a good understanding of both features is a requirement for high-performance soft magnetic materials [13]. In fact, it is well-known that both skin depth and circumferential permeability are interrelated, and in magnetic microwires of radius $a$ can be given as follows [24,42,43]:

$$
\delta_{\mathbf{m}}=\frac{1}{\sqrt{\pi \sigma \mu_{\phi} f}}
$$


where $\sigma$ and $\mu_{\phi}$ are the electrical conductivity and circumferential magnetic permeability, respectively.

In view of this, we can tackle the GMI response by drawing a general description of skin effect and magnetic anisotropy. The frequency studied in this paper covers the frequency range from 10 to $1000 \mathrm{MHz}$, in which the skin depth $\delta_{m}$ plays an important role. In panels (a) and (b) of Figures 3-5, the GMI exhibits the expected double-peak behaviour for the as-prepared sample (in the whole frequency range), which results from the induced magnetoelastic anisotropy during the fabrication. By contrast, at $100 \mathrm{MHz}$ a single-peak response is achieved by specific conventional annealing (at $T_{a n n} \geq 300{ }^{\circ} \mathrm{C}$ in Figure 3a) and stress-annealed samples ( $\sigma_{m} \leq 236 \mathrm{MPa}$ in Figures $4 \mathrm{a}$ and $5 \mathrm{a}$, respectively). This is the result of vanishing induced magnetoelastic anisotropies during their fabrication. Moreover, in annealed and stress-annealed samples (at low $\sigma_{m}$-values) the internal stresses' relaxation contributes to the axial anisotropy, and therefore, GMI response tends to be single-peak. However, in stress-annealed samples, at sufficiently high $\sigma_{m}$-values, the circumferential anisotropy becomes more relevant and double-peak behaviour is observed at $100 \mathrm{MHz}$. In addition, at $500 \mathrm{MHz}$ all the treated samples exhibit double-peak behaviour. Once more, this is straightforward explained through the frequency dependence of the skin effect in magnetic microwires (see Equation (4)), while the influence of magnetoelastic anisotropies are taken into consideration. In this sense, at relatively low frequencies, it is assumed that the current flows through the whole ferromagnetic nucleus, i.e., the skin depth is comparable to the microwire radius, i.e., $\delta_{m} \approx a[33,44]$. However, as frequencies increase, the skin depth decreases and hence the current flows closer to the surface while inducing the circumferential magnetic anisotropy near the metallic nucleus surface, which in turn becomes more relevant. For that reason, it is expected a magnetic evolution from single to double-peak behaviour in the GMI response for some of the samples considered in this study.

Regarding the key role of the skin effect in the GMI response, where $Z \sim 1 / \delta_{m}[43,44]$, in Figure 6 we show the penetration skin depth dependence of $(\Delta Z / Z)_{\max }$. The skin depth $\delta_{m}$ has been estimated through Equation (5) by considering that the real component of the measured impedance stems from the variations of the effective surface where the ac-current flows as a result of the skin effect [44-46]. This approach connects $\delta_{m}$ with the ratio $\mathrm{R}_{\mathrm{DC}} / \mathrm{R}_{\mathrm{AC}}$, as follows:

$$
\delta_{m}=a\left[1-\left(1-\frac{R_{D C}}{R_{A C}}\right)^{1 / 2}\right]
$$

where $R_{D C}$ is the dc-resistance of the wire, and $R_{A C}$ is the real component of the measured impedance at a given frequency as a function of the axially applied dc-field, and $a$ is the wire radius. The minimum skin depth $\delta_{m}^{\min }$ represented in Figure 6 is obtained from Equation (5) for each frequency in all samples.
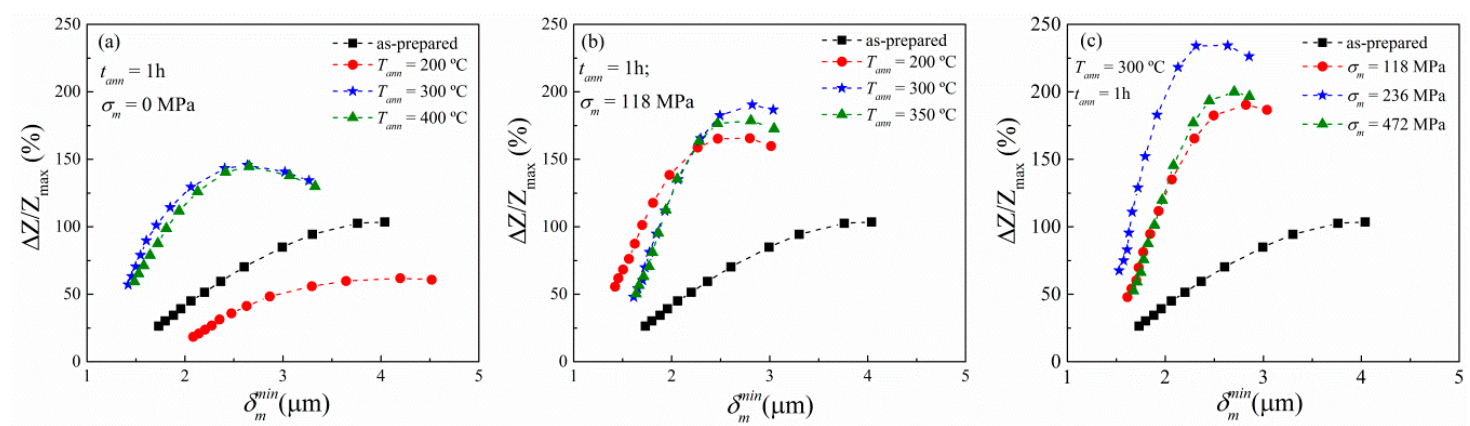

Figure 6. Minimum skin depth dependence of $(\Delta Z / Z)_{\max }$ for as-prepared and conventional annealed (a), as-prepared and stress-annealed at a fixed $\sigma_{m}$ of $118 \mathrm{MPa}$ while varying the $T_{\text {ann }}(\mathbf{b})$, and at a fixed annealing temperature while varying the applied stress (c) for the $\mathrm{Co}_{69.2} \mathrm{Fe}_{3.6} \mathrm{Ni}_{1} \mathrm{~B}_{12.5} \mathrm{Si}_{11} \mathrm{Mo}_{1.5} \mathrm{C}_{1.2}$ microwires. Dashed-lines are a guide to the eye, while full-symbols denote experimental data. The frequency dependence increases from right to left in contrast to panel (c) of Figures 3-5. 
Although, the $(\Delta Z / Z)_{\max }$ represented here redraws the same tendency as in the panel (c) of Figures $3-5$, that is, the increment (or diminishing for the conventional annealed sample at $200{ }^{\circ} \mathrm{C}$ ) in the GMI effect depending on the treatment. In Figure 6 the treatment effect on the GMI response is more clearly evident. As a general rule of thumb, the treatment efficiency is directly related to the reduction of the $\delta_{m}^{\min }$ while the GMI effect is improved. Thus, the skin depth not only gives a direct hint of the (positive/negative) influence of the treatment in the GMI response but also delimits the range of efficacy.

As mentioned above, the GMI response relies on the skin effect. Moreover, this effect only becomes relevant when $\delta_{\mathrm{m}} \lesssim \mathrm{a}$, that is, in the intermediate frequency range where the maximal $(\Delta Z / Z)_{\max }$ is expected to occur $[13,25]$. Bear in mind that $f_{\text {char }}$ is defined as the frequency at which the GMI response is maximal. Taking this into consideration along with Equation (4), we obtain the following qualitative expression for the $f_{\text {char }}[43,44]$ :

$$
f_{\text {char }}=\frac{1}{\pi \mu_{\phi} \sigma a^{2}}
$$

From Equation (6) it is inferred that the higher the circumferential magnetic permeability, the lower the $f_{\text {char }}$. This is experimentally evinced in panel (c) of Figures 3-5. Specifically, in Figure 3c the as-prepared sample exhibits the $f_{\text {char }}$ at $80 \mathrm{MHz}$, whereas the conventional annealed samples show the maximal GMI response at a higher $f_{\text {char }}$, i.e., $100 \mathrm{MHz}$ for the $T_{\text {ann }}$ at $200{ }^{\circ} \mathrm{C}$ and $150 \mathrm{MHz}$ for the $T_{\text {ann }}$ samples at 300 and $400{ }^{\circ} \mathrm{C}$. Similarly, Figures $4 \mathrm{c}$ and $5 \mathrm{c}$ display the same tendency for the $f_{\text {char }}$. In this sense, all the treated samples presented in this work show an increase in the $f_{\text {char }}$ as a result of the gradual internal-stresses relaxation that contributes to the axial anisotropy, but at the expense of the circumferential anisotropy.

Field sensitivity is represented in panel (d) of Figures 3-5. There it is noticed the positive effect the stress-annealing on field sensitivity in Figure $5 \mathrm{~d}$. This could be derived either from the induced magnetoelastic anisotropy upon stress-annealing, or even from a change in the magnetostriction coefficient. Furthermore, Figure $4 \mathrm{~d}$ shows an improvement in the sensitivity as the $T_{\text {ann }}$ rises up to a critical value of $300{ }^{\circ} \mathrm{C}$. Above this temperature, the sensitivity is reduced. We surmise this counterbalance effect as a consequence of inner stress relaxation, i.e., the annealing above $300^{\circ} \mathrm{C}$ vanish the magnetoelastic anisotropy in the microwire, which in turn is negatively reflected in both GMI response and field sensitivity.

Finally, microwires with different chemical composition and internal stresses (to a great extent related to the thickness of the glass-coating [30,31]) must present distinct GMI performance. However, similar effects of conventional annealing on the magnetic properties of Co-rich microwires of different chemical composition have already been reported, i.e., a significant magnetic hardening in various Co-based microwires with vanishing $\lambda_{\mathrm{s}}$-values has been noticed upon applying a conventional annealing [32]. Such magnetic hardening negatively affects the GMI performance [26,27,32]. In the present case, we have identified the precise route that enhances the GMI response and field sensitivity in the studied microwire with a well-established chemical composition. We anticipate that experimental dependencies concerning Co-rich microwires will be disclosed in the near future.

\section{Conclusions}

In summary, we have performed a comprehensive investigation of the GMI response and the field sensitivity by modifying the magnetoelastic anisotropies through different thermal treatments (conventional and stress-annealing) on the ferromagnetic amorphous $\mathrm{Co}_{69.2} \mathrm{Fe}_{3.6} \mathrm{Ni}_{1} \mathrm{~B}_{12.5} \mathrm{Si}_{11} \mathrm{Mo}_{1.5} \mathrm{C}_{1.2}$ glass-coated microwire. On the one hand, the findings reported reveal stress-annealing as the suitable technique to improve simultaneous and remarkably the GMI effect and the field sensitivity. In this sense, it is observed a maximum GMI response of $234 \%$ for the exciting current frequency of $100 \mathrm{MHz}$ and a maximum field sensitivity of $104 \% / O$ for the $300{ }^{\circ} \mathrm{C}$ stress-annealing sample at 236 and $472 \mathrm{MPa}$, respectively. Moreover, a significant frequency dependence of field sensitivity is attained in the stress-annealed samples. These results have been discussed in terms of the frequency dependence of 
skin depth, along with the magnetoelastic anisotropy modification. The presented outcomes can be used as a guide in further studies while deepening the knowledge to draw future lines in materials design. Hence, we evinced the stress-annealed $\mathrm{Co}_{69.2} \mathrm{Fe}_{3.6} \mathrm{Ni}_{1} \mathrm{~B}_{12.5} \mathrm{Si}_{11} \mathrm{Mo}_{1.5} \mathrm{C}_{1.2}$ glass-coated microwire as a prospective material for sensor applications.

Author Contributions: D.G.-A. and L.G.-L. wrote the manuscript; V.Z., L.G.-L. and P.C.-L. prepared and annealed the samples; M.I., V.Z., J.M.B., L.G.-L. and P.C.-L. performed the magnetic and GMI measurements; L.G.-L., D.G.-A. and A.Z. participated in the result analysis and discussion. All the authors reviewed and finalized the manuscript. All authors have read and agreed to the published version of the manuscript.

Funding: D.G.-A is founded by MAT2017-83631-C3-R. This work was also supported by Spanish MCIU under PGC2018-099530-B-C31 (MCIU/AEI/FEDER, UE) and by the Government of the Basque Country under PIBA 2018-44 project and by the University of Basque Country under the scheme of "Ayuda a Grupos Consolidados" (Ref.: GIU18/192).

Acknowledgments: Authors give thanks for technical and human support to SGIker of UPV/EHU (Medidas Magnéticas Gipuzcoa) and European funding (ERDF and ESF).

Conflicts of Interest: Authors declare no conflict of interest.

\section{References}

1. Díaz-Michelena, M. Small Magnetic Sensors for Space Applications. Sensors 2009, 9, 2271-2283. [CrossRef]

2. Nowicki, M.; Szewczyk, R. Determination of the Location and Magnetic Moment of Ferromagnetic Objects Based on the Analysis of Magnetovision Measurements. Sensors 2019, 19, 337. [CrossRef]

3. Lenz, J.; Edelstein, A.S. Magnetic Sensors and their applications. IEEE Sens. J. 2006, 6, 631-649. [CrossRef]

4. Mohri, K.; Uchiyama, T.; Panina, L.V. Recent advances of micro magnetic sensors and sensing application. Sens. Actuators A 1997, 59, 1-8. [CrossRef]

5. Kozejova, D.; Fecova, L.; Klein, P.; Sabol, R.; Hudak, R.; Sulla, I.; Mudronova, D.; Galik, J.; Varga, R. Biomedical applications of glass-coated microwires. J. Magn. Magn. Mater. 2019, 470, 2-5. [CrossRef]

6. Morales, I.; Archilla, D.; de la Presa, P.; Hernando, A.; Marin, P. Colossal heating efficiency via Eddy currents in amorphous microwires with nearly zero magnetostriction. Sci. Rep. 2020, 10, 602. [CrossRef] [PubMed]

7. Panina, L.V.; Mohri, K. Magneto-impedance effect in amorphous wires. Appl. Phys. Lett. 1994, 65, 1189-1191. [CrossRef]

8. Panina, L.V.; Mohri, K.; Uchyama, T.; Noda, M. Giant Magneto-Impedance in Co-Rich Amorphous Wires and Films. IEEE Trans. Magn. 1995, 31, 1249. [CrossRef]

9. Hauser, M.; Kraus, L.; Ripka, P. Giant magnetoimpedance sensors. IEEE Instrum. Meas. Mag. 2001, 4, 28-32. [CrossRef]

10. Ripka, P.; Vertesy, G. Sensors based on soft magnetic materials Panel discussion. J. Magn. Magn. Mater. 2000, 215, 795-799. [CrossRef]

11. Jiles, D.C. Recent advances and future directions in magnetic materials. Acta Mater. 2003, 51, 5907-5939. [CrossRef]

12. Panina, L.V.; Makhnovskiy, D.P.; Dzhumazoda, A.; Podgornaya, S.V. High Performance Soft Magnetic Materials. In Springer Series in Materials Science; Zhukov, A., Ed.; Springer International Publishing: Cham, Switzerland, 2017; p. 216. ISBN 0933-033X.

13. Kraus, L.; Vázquez, M.; Knobel, M. Giant Magnetoimpedance. In Handbook of Magnetic Materials; Elsevier: Amsterdam, The Netherlands, 2003; p. 497, ISSN: 1567-2719.

14. Pirota, K.R.; Kraus, L.; Chiriac, H.; Knobel, M. Magnetic properties and giant magnetoimpedance in CoFeSiB glass-covered microwires. J. Magn. Magn. Mater. 2000, 221, L243-L247. [CrossRef]

15. Zhukov, A.; Zhukova, V.; Blanco, J.M.; Gonzalez, J. Recent research on magnetic properties of glass-coated microwires. J. Magn. Magn. Mater. 2005, 294, 182-192. [CrossRef]

16. Mohri, K.; Uchiyama, T.; Panina, L.V.; Yamamoto, M.; Bushida, K. Recent Advances of Amorphous Wire CMOS IC Magneto-Impedance Sensors: Innovative High-Performance Micromagnetic Sensor Chip. J. Sens. 2015, 2015, 718069. [CrossRef]

17. Dufay, B.; Saez, S.; Dolabdjian, C.; Melo, L.G.C.; Yelon, A.; Ménard, D. Development of a high sensitivity Giant Magneto-Impedance magnetometer: Comparison with a commercial Flux-Gate. IEEE Trans. Magn. 2013, 49, 85. [CrossRef] 
18. Uchiyama, T.; Mohri, K.; Nakayama, S. Measurement of Spontaneous Oscillatory Magnetic Field of Guinea-Pig Smooth Muscle Preparation Using Pico-Tesla Resolution Amorphous Wire Magneto-Impedance Sensor. IEEE Trans. Magn. 2011, 47, 3070-3073. [CrossRef]

19. Chen, L.; Bao, C.C.; Yang, H.; Li, D.; Lei, C.; Wang, T.; Hu, H.Y.; He, M.; Zhou, Y.; Cui, D.X. A prototype of giant magnetoimpedance-based biosensing system for targeted detection of gastric cancer cells. Biosens. Bioelectron. 2011, 26, 3246-3253. [CrossRef]

20. Ulitovsky, A.V.; Maianski, I.M.; Avramenco, A.I. Method of Continuous Casting of Glass Coated Microwire. USSR Patent No. 128427, 15 May 1960. p. 14.

21. Zhukov, A.; Ipatov, M.; Corte-León, P.; Gonzalez-Legarreta, L.; Churyukanova, M.; Blanco, J.M.; Gonzalez, J.; Taskaev, S.; Hernando, B.; Zhukova, V. Giant magnetoimpedance in rapidly quenched materials. J. Alloy Compd. 2020, 814, 152225. [CrossRef]

22. Sandacci, S.; Makhnovskiy, D.; Panina, L.; Larin, V. Stress-dependent magnetoimpedance in Co-based amorphous wires with induced axial anisotropy for tunable microwave composites. IEEE Trans. Magn. 2005, 41, 3553-3555. [CrossRef]

23. Zhukov, A.; Talaat, A.; Churyukanova, M.; Kaloshkin, S.; Semenkova, V.; Ipatov, I.; Blanco, J.M.; Zhukova, V. Engineering of magnetic properties and GMI effect in Co-rich amorphous microwires. J. Alloy. Comp. 2016, 664, 235-241. [CrossRef]

24. Panina, L.V.; Mohri, K.; Bushida, K.; Noda, M. Giant magnetoimpedance and magnetoinductive effects in amorphous alloys. J. Appl. Phys. 1994, 76, 6198. [CrossRef]

25. Phan, M.H.; Peng, H.X. Giant magnetoimedance materials: Fundamentals and applications. Prog. Mater. Sci. 2008, 53, 323-420. [CrossRef]

26. Zhukov, A.; Ipatov, M.; Zhukova, V. Advances in Giant Magnetoimpedance of Materials. In Handbook of Magnetic Materials; Buschow, K.H.J., Ed.; Elsevier: Amsterdam, The Netherlands, 2015; Volume 24, pp. 139-236.

27. Zhukov, A.; Ipatov, M.; Corte-León, P.; González-Legarreta, L.; Blanco, J.M.; Zhukova, V. Soft magnetic microwires for sensor applications. J. Magn. Magn. Mater. 2020, 498, 166180. [CrossRef]

28. Zhukov, A.; Churyukanova, M.; Kaloshkin, S.; Sudarchikova, V.; Gudoshnikov, S.; Ipatov, M.; Talaat, A.; Blanco, J.M.; Zhukova, V. Magnetostriction of Co-Fe-based amorphous soft magnetic microwires. J. Electr. Mater. 2016, 45, 226-234. [CrossRef]

29. Konno, Y.; Mohri, K. Magnetostriction measurements for amorphous wires. IEEE Trans. Magn. 1989, 25, 3623-3625. [CrossRef]

30. Zhukov, A.; Gonzalez, J.; Torcunov, A.; Pina, E.; Prieto, M.J.; Cobeño, A.F.; Blanco, J.M.; Larin, S.; Baranov, V. Ferromagnetic resonance and Structure of Fe-based Glass-coated Microwires. J. Magn. Magn. Mater. 1999, 203, 238-240. [CrossRef]

31. Chiriac, H.; Óvári, T.-A.; Corodeanu, S.; Ababei, G. Interdomain wall in amorphous glass-coated microwires. Phys. Rev. B 2007, 76, 214433. [CrossRef]

32. Zhukova, V.; Ipatov, M.; Talaat, A.; Blanco, J.M.; Churyukanova, M.; Zhukov, A. Effect of stress annealing on magnetic properties and GMI effect of Co- and Fe-rich microwires. J. Alloys Compd. 2017, 707, 189-194. [CrossRef]

33. Gonzalez-Legarreta, L.; Corte-Leon, P.; Zhukova, V.; Ipatov, M.; Blanco, J.M.; Gonzalez, J.; Zhukov, A. Optimization of magnetic properties and GMI effect of Thin Co-rich Microwires for GMI Microsensors. Sensors 2020, 20, 1588. [CrossRef]

34. Zhukova, V.; Corte-Leon, P.; Ipatov, M.; Blanco, J.M.; González-Legarreta, L.; Zhukov, A. Development of Magnetic Microwires for Magnetic Sensor Applications. Sensors 2019, 19, 4767. [CrossRef] [PubMed]

35. Gonzalez-Legarreta LCorte-León, P.; Zhukova, V.; Ipatov, M.; Blanco, J.M.; Churyukanova, M.; Taskaev, S.; Zhukov, A. Route of magnetoimpedance and domain walls dynamics optimization in Co-based microwires. J. Alloy. Comp. 2020, 830, 154576. [CrossRef]

36. Zhukova, V.; Cobeño, A.F.; Zhukov, A.; de Arellano Lopez, A.R.; López-Pombero, S.; Blanco, J.M.; Larin, V.; Gonzalez, J. Correlation between magnetic and mechanical properties of devitrified glass-coated $\mathrm{Fe}_{71.8} \mathrm{Cu}_{1} \mathrm{Nb}_{3.1} \mathrm{Si}_{15} \mathrm{~B}_{9.1}$ microwires. J. Magn. Magn. Mater. 2002, 249, 79-84. [CrossRef]

37. Zhukova, V.; Cobeño, A.F.; Zhukov, A.; Blanco, J.M.; Puerta, S.; Gonzalez, J.; Vázquez, M. Tailoring of magnetic properties of glass coated microwires by current annealing. J. Non-Cryst. Solids 2001, 287, 31-36. [CrossRef] 
38. Zhukova, V.; Blanco, J.M.; Ipatov, M.; Churyukanova, M.; Taskaev, S.; Zhukov, A. Tailoring of magnetoimpedance effect and magnetic softness of Fe-rich glass-coated microwires by stress- annealing. Sci. Rep. 2018, 8, 3202. [CrossRef]

39. Zhukov, A.; Chichay, K.; Talaat, A.; Rodionova, V.; Blanco, J.M.; Ipatov, M.; Zhukova, V. Manipulation of magnetic properties of glass-coated microwires by annealing. J. Magn. Magn. Mater. 2015, 383, 232-236. [CrossRef]

40. Churyukanova, M.; Semenkova, V.; Kaloshkin, S.; Shuvaeva, E.; Gudoshnikov, S.; Zhukova, V.; Shchetinin, I.; Zhukov, A. Magnetostriction investigation of soft magnetic microwires. Phys. Status Solidi A 2016, 213, 363-367. [CrossRef]

41. Cobeño, A.F.; Zhukov, A.; Blanco, J.M.; Larin, V.; Gonzalez, J. Magnetoelastic sensor based on GMI of amorphous microwire. Sens. Actuators A 2001, 91, 95-98. [CrossRef]

42. Aragoneses, P.; Zhukov, A.; Gonzalez, J.; Blanco, J.M.; Dominguez, L. Effect of AC driving current on Magneto-Impedance effect. Sens. Actuators A 2000, 81, 86-90. [CrossRef]

43. Mishra, A.C.; Sahoo, T.; Srinivas, V.; Thakur, A.K. Giant magnetoimpedancee in electrodeposited CoNiFe/Cu wire: A study on thickness dependence. J. Alloys Compd. 2009, 480, 771. [CrossRef]

44. Lachowicz, H.K.; Garcia, K.L.; Kuzminski, M.; Zhukov, A.; Vazquez, M. Skin-effect and circumferential permeability in micro-wires utilized in GMI-sensors. Sens. Actuators A 2005, 119, 384-389. [CrossRef]

45. Zhukov, A.; Talaat, A.; Ipatov, M.; Granovsky, A.; Zhukova, V. Estimation of the frequency and magnetic field dependence of the skin depth in Co-rich magnetic microwires from GMI experiments. J. Sci. Adv. Mater. Devices 2016, 1, 388-392. [CrossRef]

46. Knobel, M.; Sanches, M.L.; Gomez-Polo, C.; Marin, P.; Vazquez, M.; Hernando, A. Giant magneto-impedance effect in nanostructured magnetic wires. J. Appl. Phys. 1996, 79, 1646-1654. [CrossRef]

(C) 2020 by the authors. Licensee MDPI, Basel, Switzerland. This article is an open access article distributed under the terms and conditions of the Creative Commons Attribution (CC BY) license (http://creativecommons.org/licenses/by/4.0/). 